\title{
Accelerated versus conventional corneal collagen cross-linking in the treatment of mild keratoconus: a comparative study
}

This article was published in the following Dove Press journal:

Clinical Ophthalmology

2 August 2014

Number of times this article has been viewed

\author{
Ahmed Mohamed Sherif \\ Faculty of Medicine, Cairo University, \\ Egypt
}

Aim: To evaluate the use of accelerated corneal collagen cross-linking in the management of mild and moderate progressive keratoconus in comparison with conventional cross-linking.

Patients and methods: Prospective randomized interventional case-control clinical trial. A total 25 eyes of 18 patients were randomly divided into two groups: group A included 14 eyes that received accelerated collagen cross-linking; and group B included eleven eyes that received conventional cross-linking (Dresden protocol). Cases with grade 1 and 2 keratoconus (Krumeich classification) with topographic evidence of progression were included. The uncorrected visual acuity and best spectacle-corrected visual acuity were recorded. Pentacam ${ }^{\circledR}$ corneal analysis imaging was used: The maximum and minimum keratometry and the central corneal thickness were recorded. Ocular Response Analyzer ${ }^{\circledR}$ (ORA) measurements were carried out to document the biomechanical parameters. Corneal hysteresis and corneal resistance factor were recorded. Postoperative evaluation included uncorrected visual acuity, best corrected visual acuity, and Pentacam and ORA measurements at 6 and 12 months. The follow-up period was 12 months.

Results: A progressive decrease in the flat keratometry, steep keratometry, and mean keratometry was noted throughout the follow-up period in both the accelerated and conventional groups. The improvement in keratometry was not statistically significant. The best spectacle-corrected visual acuity showed a statistically significant improvement in both groups by 1 year. The corneal hysteresis and corneal resistance factor showed nonsignificant improvement in both groups. The central corneal thickness showed a significant thinning at 6 months in both groups but approached baseline values by 12 months.

Conclusion: Accelerated corneal collagen cross-linking appears to show comparable results to conventional cross-linking in arresting the progression of mild keratoconus.

Keywords: accelerated collagen cross-linking, corneal hysteresis, corneal resistance factor, central corneal thickness

\section{Introduction}

Keratoconus $(\mathrm{KC})$ is a progressive, frequently asymmetric, noninflammatory corneal thinning disorder characterized by changes in the structure and organization of corneal collagen. The disease classically manifests in the second decade of life, when the cornea assumes an increasingly conical shape owing to its biomechanical instability. ${ }^{1}$

After initial encouraging laboratory evidence, a pilot study by Wollensak et al demonstrating successful halting of KC progression was published in 2003. The researchers utilized ultraviolet (UV)A light and riboflavin as a photosensitizer, to induce covalent cross-link bonds between collagen fibers in the corneal stroma, thereby increasing its biomechanical strength and stability. ${ }^{2}$ There have been numerous publications in the peer-reviewed literature over the last decade, with a variety
Sherif

Department of Ophthalmology,

Faculty of Medicine, Cairo University,

7 Awad Amer Street, Mohandessin,

Giza, Egypt

Tel +20 I22 I08 8299

Email asherif1975@yahoo.com 
of methodologies (retrospective, prospective uncontrolled, and randomized controlled trials), addressing the safety and efficacy of corneal collagen cross-linking (CXL) in the treatment of $\mathrm{KC}$ as well as other corneal ecstatic disorders. ${ }^{3}$ These studies have provided sufficient evidence that CXL is successful in slowing or halting $\mathrm{KC}$ progression and may even demonstrate visual, topographic, and aberrometric improvement through induced corneal flattening and reduction in irregular astigmatism. ${ }^{3}$ Importantly, medium- and long-term studies have validated an excellent safety profile for standard CXL (epithelium-off method). ${ }^{4}$ In the Dresden protocol, a 30-minute instillation of riboflavin is followed by 30 minutes of $365 \mathrm{~nm}$ UVA illumination at $3 \mathrm{~mW} / \mathrm{cm}^{2}$ (equivalent to a $5.4 \mathrm{~J} / \mathrm{cm}^{2}$ dose).

The technique of accelerated CXL was recently introduced. This aims to: reduce patient discomfort; to achieve more effective time management; and to avoid the excessive corneal dehydration and thinning that can occur during the 30-minute period and that may lead to deeper penetration and riboflavin/UVA toxicity in the corneal endothelium. ${ }^{5}$ The idea behind CXL is to shorten the illumination time by increasing the illumination intensity, while maintaining a constant radiant exposure of $5.4 \mathrm{~J} / \mathrm{cm}^{2}$, according to the Bunsen-Roscoe law of reciprocity. ${ }^{6}$ With the

A

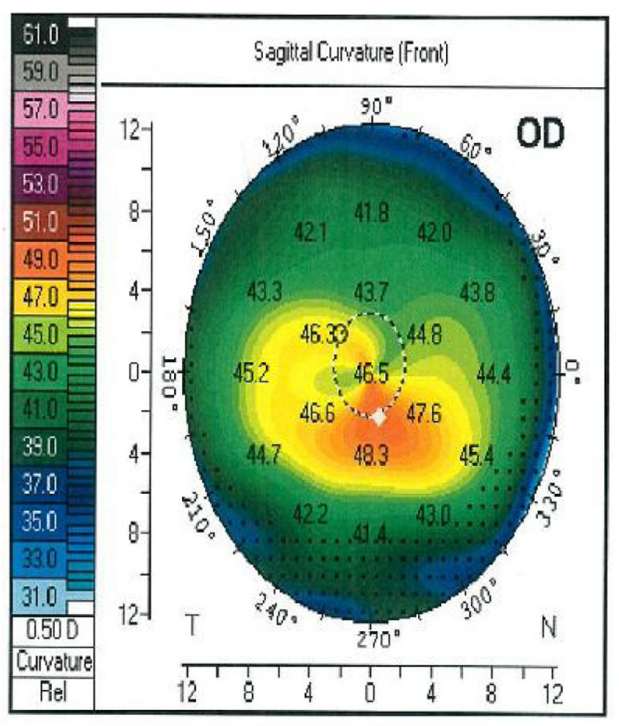

B

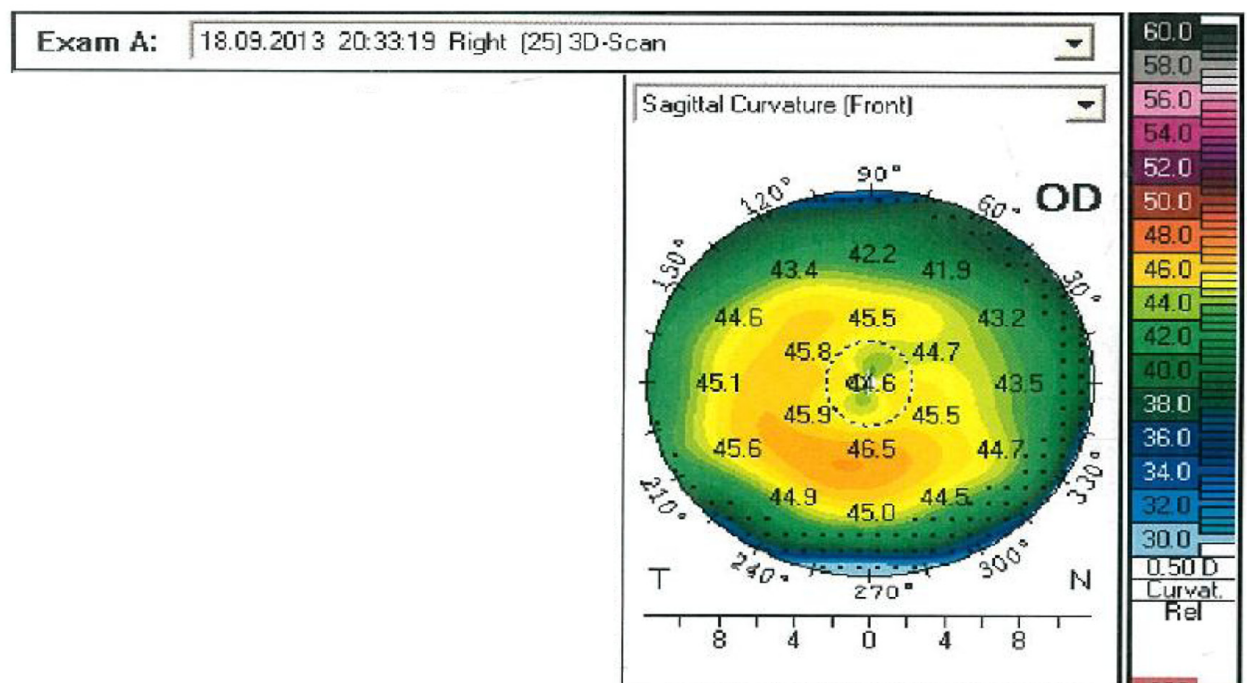

Figure I (A) Pentacam ${ }^{\circledast}$ (HR Premium; Oculus Optikgeräte GmbH, Wetzlar, Germany) image before accelerated cross-linking. (B) Pentacam image 9 months after accelerated cross-linking.

Abbreviations: OD, oculus dexter (right eye); 3D, three dimensional. 


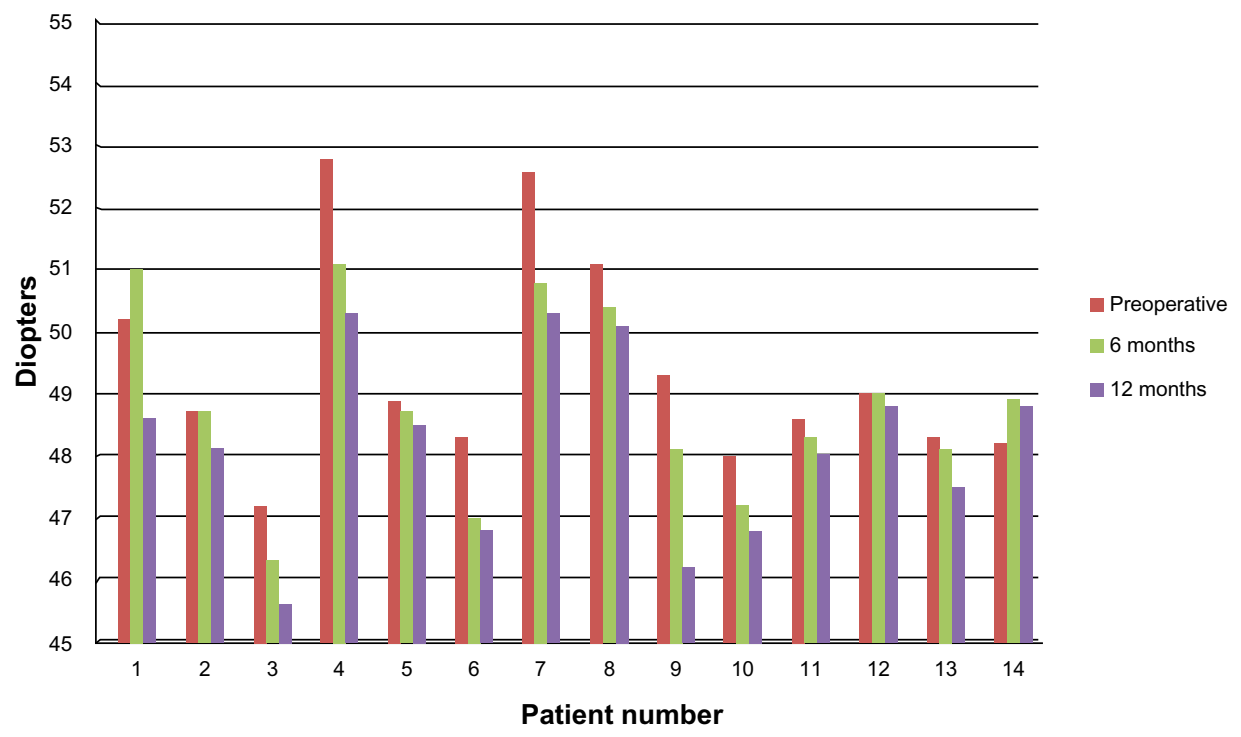

Figure $2 \mathrm{~K}_{\max }$ changes in the accelerated cross-linking group. Abbreviation: $\mathrm{K}_{\max }$, steep keratometry.

recent advances in UV delivery systems, intensities of up to $45 \mathrm{~mW} / \mathrm{cm}^{2}$ are available, which permit a possible reduction of UV exposure time down to 2 minutes. ${ }^{7,8}$ It has been shown with confocal microscopy that accelerated CXL leads to anterior stromal changes similar to those produced by conventional CXL, lasting for up to 6 months. ${ }^{9}$

The aim of this study was to evaluate the use of accelerated CXL in the management of progressive $\mathrm{KC}$, in comparison with conventional CXL.

\section{Patients and methods}

The study was a prospective, randomized interventional clinical study in which 25 eyes of 18 patients were randomly divided into two groups: group A, with 14 eyes, received accelerated CXL; and group B, with eleven eyes, received standard CXL (Dresden protocol). ${ }^{2}$

\section{Inclusion criteria}

The inclusion criteria included grade 1 and $2 \mathrm{KC}$ (Krumeich classification), ${ }^{10}$ with topographic evidence of progression as defined by: an increase of $1.0 \mathrm{D}$ or more in the steepest keratometry $(\mathrm{K})$; an increase of $1.0 \mathrm{D}$ or more in the manifest cylinder; or an increase of $0.5 \mathrm{D}$ or more in the manifest refraction spherical equivalent over a 6-month period. A minimum pachymetry of $400 \mu \mathrm{m}$ at the thinnest corneal location was obligatory.

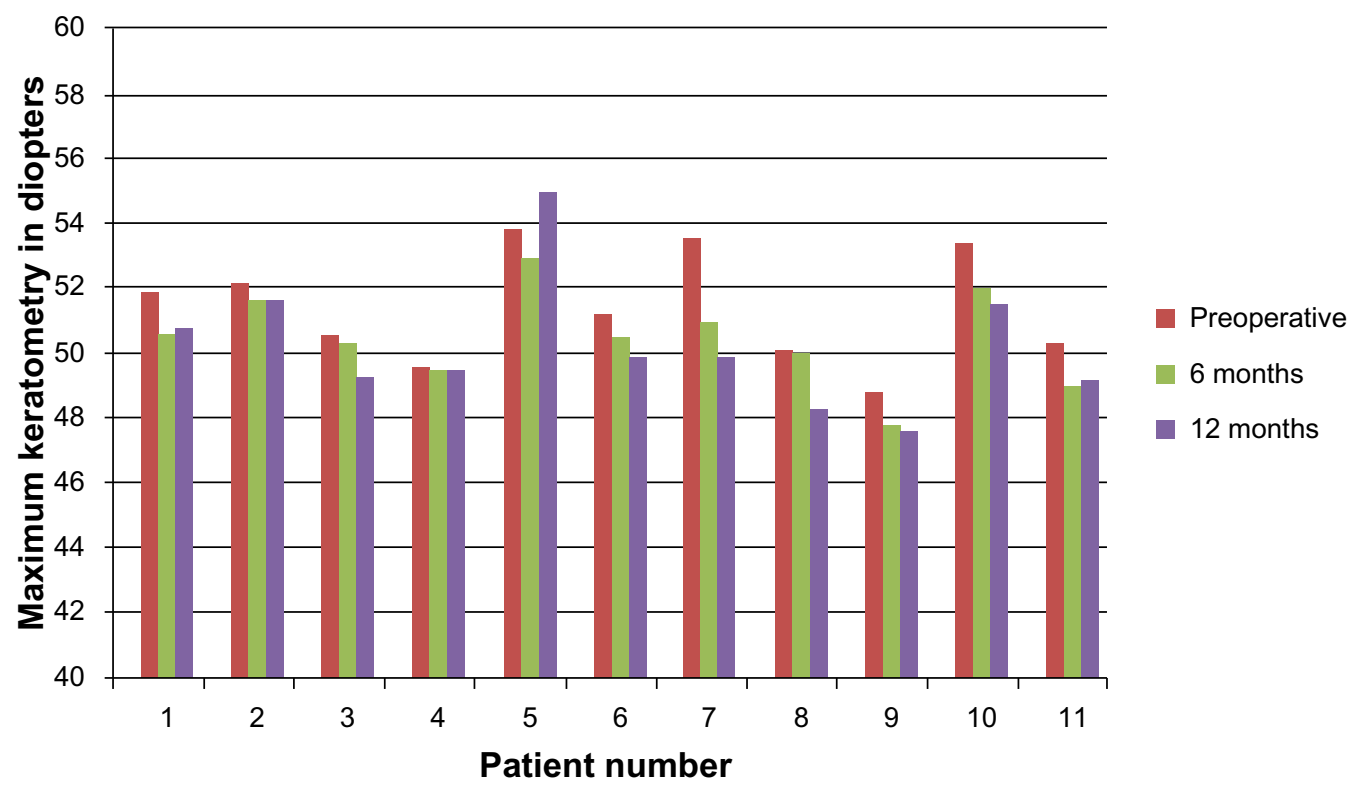

Figure $3 \mathrm{~K}_{\max }$ changes in the conventional cross-linking group. Abbreviation: $\mathrm{K}_{\max }$, steep keratometry. 
Table I Summary of the results of the accelerated and conventional cross-linking groups

\begin{tabular}{lcc}
\hline & $\begin{array}{l}\text { Accelerated } \\
\text { group }\end{array}$ & $\begin{array}{l}\text { Conventional } \\
\text { group }\end{array}$ \\
\hline Preoperative visual acuity & $0.48 \pm 0.17$ & $0.49 \pm 0.19$ \\
Visual acuity at 6 months & $0.5 \pm 0.2$ & $0.57 \pm 0.19$ \\
Visual acuity at I2 months & $0.61 \pm 0.15$ & $0.64 \pm 0.16$ \\
Preoperative maximum & $49.43 \pm 1.63$ & $51.4 \pm 1.69$ \\
keratometry (D) & & \\
Maximum keratometry & $48.83 \pm 1.52$ & $50.47 \pm 1.42$ \\
at 6 months $(D)$ & & \\
Maximum keratometry & $48.2 \pm 1.43$ & $50.24 \pm 2$ \\
at I2 months $(D)$ & $484.57 \pm 19.45$ & $464.18 \pm 29.15$ \\
Preoperative central & & \\
corneal thickness $(\mu \mathrm{m})$ & $458.42 \pm 18.32$ & $438.27 \pm 21.77$ \\
Central corneal thickness & & \\
at 6 months $(\mu \mathrm{m})$ & $469.64 \pm 20$ & $451.73 \pm 24.39$ \\
Central corneal thickness & & \\
at I2 months $(\mu \mathrm{m})$ & &
\end{tabular}

Abbreviation: $\mathrm{D}$, diopter.

Cases with apical opacity or hydrops were excluded. A history of herpetic corneal disease or previous ocular surgery were considered contraindications.

\section{Preoperative assessment}

A slit lamp evaluation, including applanation tonometry and dilated fundoscopy, using a $+90 \mathrm{D}$ lens, was performed. Manifest and cycloplegic refraction were recorded. Next, a corneal analysis (Pentacam ${ }^{\circledR}$ HR Premium; Oculus Optikgeräte $\mathrm{GmbH}$, Wetzlar, Germany) was performed, and the topographic maps were studied. To study the biomechanical parameters of the cornea, Ocular Response Analyzer ${ }^{\circledR}$ (ORA) (Reichert Technologies, Inc., Depew, NY, USA) measurements were obtained: corneal hysteresis $(\mathrm{CH})$ and corneal resistance factor (CRF) were recorded.

The study was conducted according to the tenets of the Declaration of Helsinki. The Rowad Cornea Center ethical committee approved the study. The nature of the treatment was explained to all patients, and a written informed consent was obtained in all cases.

Statistical analysis was performed using Statistical Package for Social Science Version 17 (SPSS Inc., Chicago, IL, USA) version 16.0. The independent paired $t$-test was used. A $P$-value $\leq 0.05$ was considered to be statistically significant.

\section{Operative steps}

All cases were performed at Rowad Cornea Center, Giza, Egypt.

After topical anesthesia, the corneal epithelium was scraped using a crescent knife, and corneal thickness was measured using contact pachymetry (PacScan ${ }^{\mathrm{TM}} 300 \mathrm{P}$; Sonomed Escalon ${ }^{\mathrm{TM}}$, Wayne, PA, USA).

In group $\mathrm{A}$, a drop of riboflavin $0.1 \%$ dextran solution (VibeX ${ }^{\mathrm{TM}}$; Avedro, Inc., Waltham, MA, USA) was applied every 2 minutes for 30 minutes, followed by exposure to UV light $\left(370 \mathrm{~nm}, 30 \mathrm{~mW} / \mathrm{cm}^{2}\right)$ for 4 minutes and 20 seconds using the $\mathrm{KXL}^{\circledR}$ system (Avedro, Inc.) during which riboflavin was not applied. In the group B patients, a drop of riboflavin $0.1 \%$ solution (VibeX) was applied every 2 minutes for 30 minutes, followed by exposure to UV rays (370 nm, 3 mW/cm²) (UV-X ${ }^{\mathrm{TM}}$; Peschke Meditrade GmbH, Hünenberg, Switzerland) for 30 minutes during which riboflavin was applied every 2 minutes.

Care was taken to protect the limbus from inadvertent UV exposure in both groups. Finally, a bandage contact lens was applied, and a topical antibiotic (Zymar; Allergan, Inc., Irvine, CA, USA) and a topical nonsteroidal anti-inflammatory drug Nevanac (Alcon Laboratories, Inc., Fort Worth, TX, USA) was prescribed. Topical steroids were started after complete epithelial healing and were tapered over 1 month.

\section{Postoperative evaluation}

Slit lamp examination was performed at 1 day, 4 days, 1 month, 6 months, and at 12 months postsurgery. Uncorrected visual acuity (UCVA), best corrected visual acuity (BCVA), and manifest refraction were measured at 6 months and 12 months. Pentacam corneal analysis and ORA measurements were performed at 6 and 12 months. The entire follow-up period lasted 12 months.

\section{Results}

The study included 25 eyes of 18 patients (eleven males and seven females) (Figures 1-3).

The accelerated CXL group included 14 eyes of ten patients (five females and five males). The mean age of this group was $21.58 \pm 5.78$ years. In the accelerated CXL group, the mean preoperative flat keratometry $\left(\mathrm{K}_{\min }\right)$ was $47.02 \pm 1.88$ $\mathrm{D}$ and was reduced to $46.95 \pm 2.06 \mathrm{D}$ at 6 months after surgery. The reduction was not statistically significant $(P=0.46)$. At 12 months, it declined further, to $46.6 \pm 1.89 \mathrm{D}$; however, the reduction from baseline values was not statistically significant $(P=0.28)$. The mean preoperative steep keratometry $\left(\mathrm{K}_{\max }\right)$ was $49.29 \pm 1.73 \mathrm{D}$ and was reduced to $48.83 \pm 1.52 \mathrm{D}$ 6 months after surgery $(P=0.16)$; at 12 months postsurgery, it was further reduced to $48.2 \pm 1.43 \mathrm{D}$. This was a statistically significant reduction $(P=0.022)$. The reduction in the $\mathrm{K}_{\max }$ of the accelerated CXL group in this study was $1.09 \pm 0.85 \mathrm{D}$ and was comparable with the reduction of $\mathrm{K}_{\max }$ in the conventional 
group $(0.84 \pm 0.54 \mathrm{D})$. The difference between both groups was not statistically significant $(P=0.38)$.

The mean central corneal thickness (CCT) showed a statistically significant reduction from baseline $(484.57 \pm 19.45 \mu \mathrm{m}), 6$ months after accelerated CXL $(458.42 \pm 18.32 \mu \mathrm{m})(P=0.0006)$. At 12 months, it was increased to $469.64 \pm 20 \mu \mathrm{m}$. However, this was still significantly reduced from the baseline value $(P=0.028)$.

The mean preoperative BCVA was $0.48 \pm 0.17$ (decimal scale), which improved to $0.5 \pm 0.2$ at 6 months after surgery $(P=0.38)$. It continued to improve 1 year after surgery $(0.61 \pm 0.15)$, and the difference was statistically significant compared with baseline values $(P=0.015)$.

ORA measurements showed a slight change in $\mathrm{CH}$ values in the accelerated CXL group over a 12-months period: $7.83 \pm 0.54 \mathrm{mmHg}$ at baseline, $7.79 \pm 0.53 \mathrm{mmHg}$ at 6 months $(P=0.43)$, and $7.79 \pm 0.52 \mathrm{mmHg}$ at 12 months $(P=0.41)$.

CRF values improved from $6.86 \pm 0.48 \mathrm{mmHg}$ preoperatively to $7.03 \pm 0.72 \mathrm{mmHg}$ at 6 months and to $7.16 \pm 0.88 \mathrm{mmHg}$ at 12 months ( $P=0.23$ and $P=0.13$, respectively).

In the conventional group, which included eleven eyes of eight patients (six males and two females), the mean age was $23.64 \pm 4.03$ years. In the conventional group, the mean $\mathrm{K}_{\min }$ dropped from $48.29 \pm 1.68 \mathrm{D}$ to $47.99 \pm 1.58 \mathrm{D}$ at 6 months $(P=0.29)$ and to $46.37 \pm 2.28$ at 12 months $(P=0.17)$. The mean $\mathrm{K}_{\max }$ dropped from $51.4 \pm 1.69$ to $50.47 \pm 1.42$ $\mathrm{D}$ at 6 months $(P=0.13)$ and to $50.24 \pm 2 \mathrm{D}$ at 12 months $(P=0.099)$.

The CCT showed a significant reduction, from $464.18 \pm 29.15 \mu \mathrm{m}$ to $438.27 \pm 21.77 \mu \mathrm{m}$ at 6 months postCXL ( $P=0.01)$; it gradually improved, to $451.73 \pm 24.39 \mu \mathrm{m}$, which was not a statistically significant difference from the baseline values $(P=0.14)$.

The best spectacle-corrected visual acuity (BSCVA) was $0.49 \pm 0.19$ at baseline and improved to $0.57 \pm 0.19$ at 6 months. The improvement was not statistically significant $(P=0.16)$ at 12 months after surgery; the BSCVA continued to improve, to $0.64 \pm 0.16$. This improvement was statistically significant $(P=0.03)$.

$\mathrm{CH}$ values were $8.31 \pm 0.74 \mathrm{mmHg}$ at baseline and improved to $8.37 \pm 0.74 \mathrm{mmHg}$ at 6 months and to $8.54 \pm 0.69$ at 12 months. The changes were not statistically significant ( $P=0.42$ and $P=0.23$, respectively).

The CRF values showed little changes throughout the follow-up period: $7.15 \pm 1.07 \mathrm{mmHg}$ at baseline, $7.49 \pm 1.05 \mathrm{mmHg}$ at 6 months, and $7.68 \pm 1.54 \mathrm{mmHg}$ at 12 months. The changes were not statistically significant ( $P=0.23$ and $P=0.18$, respectively).
When both groups were compared regarding the amount of reduction in $\mathrm{K}_{\max }$, there was no statistically significant difference at 6 months $(P=0.23)$ and 1 year $(P=0.22)$. The same applied to the change in the $\mathrm{CH}$ and CRF: $P=0.38$ at 6 months and $P=0.27$ at 12 months for the former, and $P=0.54$ and $P=0.83$, respectively, for the latter. The CCT changes were comparable in both groups at 6 months $(P=0.99)$ and at 12 months $(P=0.66)$ (Table 1).

\section{Complications}

None of the cases in both groups lost lines of BCVA. In all cases of both groups, complete epithelialization was achieved by 4 days. No cases of infections or melting were observed in both groups. Haze was evaluated using slit lamp biomicroscopy according to the Fantes anterior stromal haze scale. ${ }^{11}$ Trace haze (grade 1) was observed in eight cases, and mild haze (grade 2) was observed during the first postoperative month in two cases of the accelerated CXL group, while in the conventional group, eight cases had trace haze, and two cases had mild haze during the same period. One case in the conventional group developed severe central haze (grade 4), which decreased gradually over the following months; at 1-year postsurgery, the patient still had a faint anterior stromal opacity, with no loss of UCVA or BSCVA. All other cases of haze in both groups resolved with topical steroids.

\section{Discussion}

CXL is one of the interesting topics in corneal surgery, with several recent modifications of the original Dresden protocol under investigation. Accelerated CXL is one of the exciting modifications of the original technique but with few published results. ${ }^{6-9}$ It has been shown that CXL leads to stabilization of $\mathrm{KC}$, with flattening of topographic keratometry and improvement of visual acuity in many cases. ${ }^{12}$

The reduction in both groups was less than the results reported by Greenstein and Hersh ${ }^{13}(1.7 \mathrm{D})$ or Caporossi et a ${ }^{14}$ (2.1 D) but were comparable to the results of Derakhshan et $\mathrm{al}^{15}(0.65)$ and Vinciguerra et $\mathrm{al}^{16}(1.1 \mathrm{D})$. The explanation is that the former two groups included more advanced cases of $\mathrm{KC}$, while the latter two groups included mild- to moderate-KC cases as the amount of flattening by CXL is directly proportional to the steepness of the cornea. ${ }^{17}$

In our study, the accelerated CXL group showed a significant reduction of the CCT 6 months after surgery, which gradually increased afterwards. A similar behavior was observed in the conventional CXL group and is similar to previous reports. The early thinning may be attributed to the compression of collagen fibrils or keratocyte apoptosis, 
among other theories. ${ }^{18}$ The cause of the later thickening between 6 and 12 months is still unclear. ${ }^{18}$

Both groups showed a statistically significant improvement in BSCVA, 1 year after surgery. Similar improvement has been reported in several studies. ${ }^{3,13-17}$

$\mathrm{CH}$ and $\mathrm{CRF}$ values showed a slight improvement in both groups that was not statistically significant. Similar results were reported by Sedaghat et al. ${ }^{19}$

Regarding complications, transient haze was noted in $71 \%$ of the accelerated CXL cases and in $91 \%$ of the conventional group. One case in the conventional CXL group developed severe anterior stromal haze (grade 4) that decreased gradually with topical steroids; at 12-months postoperative, an opacity was still visible (grade 2). This finding is consistent with other previous reports. ${ }^{20,21}$

We conclude that accelerated CXL shows comparable results with conventional CXL in arresting the progression of mild KC. Further results, with a longer follow-up period and larger sample sizes, are needed to validate this finding.

\section{Disclosure}

The author reports no conflicts of interest in this work.

\section{References}

1. Zadnik K, Steger-May K, Fink BA, et al; CLEK Study Group. Collaborative longitudinal evaluation of keratoconus. Between-eye asymmetry in keratoconus. Cornea. 2002;21(7):671-679.

2. Wollensak G, Spoerl E, Seiler T. Stress-strain measurements of human and porcine corneas after riboflavin-ultraviolet-A-induced cross-linking. J Cataract Refract Surg. 2003;29(9):1780-1785.

3. Caporossi A, Mazzotta C, Baiocchi S, Caporossi T. Long-term results of riboflavin ultraviolet a corneal collagen cross-linking for keratoconus in Italy: the Siena eye cross study. Am J Ophthalmol. 2010;149(4): 585-593.

4. Kankariya VP, Kymionis GD, Diakonis VF, Yoo SH. Management of pediatric keratoconus - evolving role of corneal collagen cross-linking: an update. Indian J Ophthalmol. 2013;61(8):435-440.

5. Holopainen JM, Krootila K. Transient corneal thinning in eyes undergoing corneal cross-linking. Am J Ophthalmol. 2011;152(4):533-536.

6. Mrochen M. Current status of accelerated corneal cross-linking. Indian J Ophthalmol. 2013;61(8):428-429.
7. Celik HU, Alagöz N, Yildirim Y, et al. Accelerated corneal crosslinking concurrent with laser in situ keratomileusis. J Cataract Refract Surg. 2012;38(8):1424-1431.

8. Schumacher S, Oeftiger L, Mrochen M. Equivalence of biomechanical changes induced by rapid and standard corneal cross-linking, using riboflavin and ultraviolet radiation. Invest Ophthalmol Vis Sci. 2011;52(12):9048-9052.

9. Touboul D, Efron N, Smadja D, Praud D, Malet F, Colin J. Corneal confocal microscopy following conventional, transepithelial, and accelerated corneal collagen cross-linking procedures for keratoconus. J Refract Surg. 2012;28(11):769-776.

10. Krumeich JH, Daniel J, Knülle A. Live-epikeratophakia for keratoconus. $J$ Cataract Refract Surg. 1998;24(4):456-463.

11. Fantes FE, Hanna KD, Waring GO 3rd, Pouliquen Y, Thompson KP, Savoldelli M. Wound healing after excimer laser keratomileusis (photorefractive keratectomy) in monkeys. Arch Ophthalmol. 1990;108(5): 665-675.

12. Koller T, Iseli HP, Hafezi F, Vinciguerra P, Seiler T. Scheimpflug imaging of corneas after collagen cross-linking. Cornea. 2009;28(5): $510-515$.

13. Greenstein SA, Hersh PS. Characteristics influencing outcomes of corneal collagen crosslinking for keratoconus and ectasia: implications for patient selection. J Cataract Refract Surg. 2013;39(8): 1133-1140.

14. Caporossi A, Baiocchi S, Mazzotta C, Traversi C, Caporossi T. Parasurgical therapy for keratoconus by riboflavin-ultraviolet type A rays induced cross-linking of corneal collagen: preliminary refractive results in an Italian study. J Cataract Refract Surg. 2006;32(5): 837-845.

15. Derakhshan A, Shandiz JH, Ahadi M, Daneshvar R, Esmaily H. Short-term outcomes of collagen crosslinking for early keratoconus. J Ophthalmic Vis Res. 2011;6(3):155-159.

16. Vinciguerra P, Albé E, Frueh BE, Trazza S, Epstein D. Two-year corneal cross-linking results in patients younger than 18 years with documented progressive keratoconus. Am J Ophthalmol. 2012;154(3):520-526.

17. Sloot F, Soeters N, van der Valk R, Tahzib NG. Effective corneal collagen crosslinking in advanced cases of progressive keratoconus. J Cataract Refract Surg. 2013;39(8):1141-1145.

18. Greenstein SA, Shah VP, Fry KL, Hersh PS. Corneal thickness changes after corneal collagen crosslinking for keratoconus and corneal ectasia: one-year results. J Cataract Refract Surg. 2011;37(4):691-700.

19. Sedaghat M, Naderi M, Zarei-Ghanavati M. Biomechanical parameters of the cornea after collagen crosslinking measured by waveform analysis. J Cataract Refract Surg. 2010;36(10):1728-1731.

20. Raiskup F, Hoyer A, Spoerl E. Permanent corneal haze after riboflavin-UVA-induced cross-linking in keratoconus. J Refract Surg. 2009;25(9):S824-S828.

21. Mazzotta C, Balestrazzi A, Baiocchi S, Traversi C, Caporossi A. Stromal haze after combined riboflavin-UVA corneal collagen cross-linking in keratoconus: in vivo confocal microscopic evaluation. Clin Experiment Ophthalmol. 2007;35(6):580-582.
Clinical Ophthalmology

\section{Publish your work in this journal}

Clinical Ophthalmology is an international, peer-reviewed journal covering all subspecialties within ophthalmology. Key topics include: Optometry; Visual science; Pharmacology and drug therapy in eye diseases; Basic Sciences; Primary and Secondary eye care; Patient Safety and Quality of Care Improvements. This journal is indexed on Submit your manuscript here: http://www.dovepress.com/clinical-ophthalmology-journal

\section{Dovepress}

PubMed Central and CAS, and is the official journal of The Society of Clinical Ophthalmology (SCO). The manuscript management system is completely online and includes a very quick and fair peer-review system, which is all easy to use. Visit http://www.dovepress.com/ testimonials.php to read real quotes from published authors. 